\title{
TRANSPOSITION ALGEBRAS
}

\author{
J. I. HALL
}

Dedicated to Professor Robert L. Griess, Jr., on the occasion of his $71^{\text {st }}$ birthday

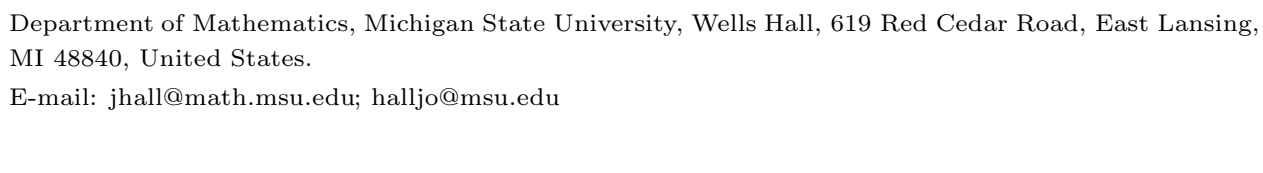

The symbiotic relationship between groups and algebras goes back at least to Sophus Lie, who introduced Lie algebras to support the study of Lie groups. Later the classification of finite dimensional, complex, semisimple Lie algebras was in turn reduced (primarily by Weyl) to the classification of finite groups generated by Euclidean reflections.

The topics discussed here had a similar start. Algebras were introduced to aid in the construction of finite simple groups, particularly those that are sporadic. These algebras then found a broader context, and there groups were reintroduced to aid in construction and classification.

Twenty years ago, Masahiko Miyamoto [40] observed that many OZ vertex operator algebras admit automorphisms canonically associated with the idempotents of the associated Griess algebra. Soon after that, Bob Griess pointed out to me that Miyamoto and others were finding use for results of mine about 3-transposition groups [10, 19, 20].

Similar algebra automorphisms have been found in other circumstances. In each instance a central question is: what automorphism groups can be generated by such elements? This survey paper॥ examines such results, particularly in the case where the automorphisms belong to a normal set of 3 -transpositions.

Received June 20, 2017 and in revised form January 24, 2018.

AMS Subject Classification: Primary: 20D05. Secondary: 17A99, 17 B69.

Key words and phrases: Sporadic simple group, 3-transposition, Lie algebra, axial algebra.

${ }^{1}$ This paper is a corrected, revised, and expanded version of my talk given at the Taipei conference honoring Bob Griess in August 2016. 


\section{Some Sporadic Groups and Algebras}

The earliest sporadic finite simple groups were constructed as permutation groups. In the mid-19 ${ }^{\text {th }}$ century, Mathieu discovered and constructed the first five sporadic groups as multiply-transitive groups of small degree. The next examples, discovered in the 1960's, were constructed as permutation groups of degree large enough to need careful computer calculation. Their construction and analysis was aided by the fact that many of the permutation representations had rank 3 [27]; that is, they were transitive with the stabilizer of a point having exactly three orbits. Thus the permutation representation carried extra structure - in this case a graph - that admitted the group as automorphisms.

Bernd Fischer [11] constructed three sporadic groups as 3-transposition groups. As such they are rank 3 permutation groups, acting by conjugation on the 3-transposition class. We shall see below that there is extra geometric structure on these representations.

From our point of view, the next major step was Simon Norton's 1976 thesis (published as [41]) in which he constructed a conjectured 3-fold cover of the largest Fischer group $F i_{24}^{\prime}$ as the automorphism group of a commutative, nonassociative algebra $N^{\natural}$ of degree 783. Again, this arose as extra structure coming from the permutation representation on the $3 \times 306936$ transpositions. From the character table, Norton observed that a 783 dimensional constituent of the permutation representation occurs as a summand in its own symmetric square. That is, the constituent admits the structure of a commutative algebra on which the group acts. Norton gave a construction by hand of this algebra, which not only proved the existence of the cover but also allowed relatively easy calculation within the group (in contrast to viewing it as a permutation group of degree nearly a million).

Soon after this, Norton observed from hypothetical character values of the conjectured Monster simple group $\mathbb{M}$ that the group should act on a commutative algebra of degree 196883 possessing an invariant bilinear form. In a tour de force, Bob Griess gave a computer-free construction of this algebra and so of $\mathbb{M}$. More precisely, Griess [16, 17] constructed an algebra extension $196884=1+196883$ having the additional property that the subalgebra fixed by $\mathrm{O}_{2}(C)$ is the Jordan algebra of symmetric $24 \times 24$ matrices. Here 
$C$ is the group of module automorphisms from which Griess constructed the algebra, thereby revealing $C$ to be the centralizer in $\mathbb{M}$ of a $2 B$ involution.

The Monster and its existence gave birth to large amounts of interesting and difficult questions and work. Up to this point we have discussed algebras in aid of groups, but now we see the other direction as well. Of particular note is the Borcherds-Frenkel-Lepowsky-Meurman [2, 12, 13] construction of a vertex algebra $V^{\natural}$ that admits $\mathbb{M}$ and naturally embeds the 196883 algebra.

Miyamoto [40] viewed $V^{\natural}$ in a more general context. A vertex operator algebra is a type of $\mathbb{Z}$-graded vertex algebra. Following Frenkel, Lepowsky, and Meurman [13, §10.3], Miyamoto observed that for any OZ vertex operator algebra $V$ (that is, $V_{<0}=0=V_{1}$ and $\operatorname{dim} V_{0}=1$ ) the weight 2 piece $V_{2}$ naturally admits a commutative algebra, and he named these Griess algebras. The piece $V_{2}$ contains exceptional elements (conformal vectors) that correspond to idempotents of the Griess algebra. Furthermore Miyamoto noted that in many situations these idempotents induce automorphisms of order 2 (involutions) on $V$ and $V_{2}$.

In the motivating special case $V=V^{\natural}$ the algebra $V_{2}=V_{2}^{\natural}$ is the Conway-Griess-Norton real algebra $M^{\natural}$, an extension $196884^{\natural}=1+196883$ that is a deformation of Griess' algebra 196884 and was studied by Conway [7. The conformal vectors/idempotents of $M^{\natural}$ are Conway's axes, and the Miyamoto involutions are precisely the $2 A$ involutions of $\mathbb{M}$. The algebra $M^{\natural}$ is a compact Griess algebra, by which we mean that it arises from a real vertex operator algebra and admits an invariant positive definite form. ॥ $^{1}$

\section{Norton Algebras}

Some group theorists find vertex operator algebras too bulky and confusing for their purposes. There have been efforts to separate a large part of the group theoretic content from that broader context. Ivanov [28] isolated several properties of the commutative algebra $M^{\natural}$ and its Miyamoto involutions to define a class of algebras admitting prescribed automorphisms, which he called Majorana involutions. Hall, Rehren, and Shpectorov [22, 23] in turn

\footnotetext{
${ }^{2}$ As the helpful referee pointed out, while many vertex operator algebras admit invariant forms, this may not be the case - especially for positive definite forms. The circumstances under which a complex vertex operator algebra admits an invariant symmetric bilinear form were investigated by $\mathrm{Li}[35]$.
} 
put these into the broader category of axial algebras, where the existence of Miyamoto involutions depends upon the presence of fusion (multiplication) rules resembling those of the Griess algebras of Miyamoto.

We will get to axial algebras below, but now we return to Norton's original constructions and motivations.

As already mentioned, Simon Norton in [41] described his construction of a 783-dimensional complex algebra $N^{\natural}$ for $3 \cdot F i_{24}^{\prime}$. The algebra is a quotient (constituent) of the (conjectured) permutation representation on $3 \times 306936$ transpositions. Norton went on in that paper to describe several similar algebra-plus-group constructions, including the 196883 algebra for the Monster. He next said:

The term "Norton algebra" was coined by J.H. Conway to describe a generic class of algebra on a small constituent of a natural permutation representation, but no definition has been found that includes these cases without being far too general.

This remains true.

The only precise definition of a Norton algebra in use today sits in an even broader context than the one described by Norton, that of association schemes - generalizations of the centralizer algebras for transitive permutation groups. The eigenspace decomposition of an association scheme generalizes the constituent decomposition of the centralizer algebra and similarly admits a natural algebra structure. Cameron, Goethals, and Seidel [5] defined a Norton algebra to be such an eigenspace with multiplication given by projection of the decomposition product. This admits numerous examples. For instance, motivated by Scott's Krein Condition [48], Cameron, Goethals, and Seidel proved that essentially every rank 3 permutation representation admits a nontrivial Norton algebra as defined. That is, at least one of the nontrivial irreducible constituents of the permutation representation occurs with positive multiplicity in its own symmetric square.

Norton preferred a narrower field. He went on to say:

However, in our special case, the present argument proves the existence of the algebra in a particularly simple manner. 
We interpret this to mean that in the algebra, as constructed by Norton, the multiplication constants with respect to the canonical spanning set are described simply. With this in mind, we offer another too-general definition:

Definition 2.1. A local Norton ( LN) algebra $(A, \mathcal{P})$ over $\mathbb{F}$ is an $\mathbb{F}$-algebra $A$ spanned by a subset $\mathcal{P}$ of vectors, with the following properties:

(i) for $x \in \mathcal{P}, x^{2}=\epsilon x$ for a fixed $\epsilon \in \mathbb{F}$;

(ii) for each $a, b \in \mathcal{P}$ the 2 -generated algebra $\ell_{a, b}=\langle a, b\rangle$ has isomorphism type from $\mathcal{A}$, a given set of $\mathbb{F}$-algebra isomorphism classes.

We may refer to $A$ as the algebra with the generating set $\mathcal{P}$ to be understood from the context.

The vectors of $\mathcal{P}$ (or, at times, the 1-spaces they span) will be called points. The 2-generated spaces of $\mathcal{L}=\left\{\ell_{a, b} \mid a, b \in \mathcal{P}\right\}$ are then lines. If $\epsilon \neq 0$ then the elements $\frac{1}{\epsilon} p$ for $p \in \mathcal{P}$ are idempotents in an algebra over $\mathbb{F}$. Thus we often choose $\epsilon$ to be 0 or 1 , although there are situations where other choices are appropriate [7, 40, 36, 37].

Under the definition $\ell_{a, b}=\ell_{b, a}$. All the examples we discuss are commutative or skew.

For a permutation constituent algebra such as Norton's $N^{\natural}$, the set $\mathcal{P}$ is the image of the set being permuted. In the examples discussed by Norton each point generates a 1-dimensional subalgebra. The parameter $\epsilon$ is then constant since the automorphism group induced by the permutation group is transitive on $\mathcal{P}$. The set $\mathcal{A}$ of isomorphism classes for 2 -generated algebras will be assumed small, corresponding to the permutation group having small rank. For $N^{\natural}$ the set $\mathcal{A}$ has size five.

The description of the algebra is local, in that the requirements only discuss 1- and 2-generated subalgebras. There are usually other global consequences or assumptions:

Definition 2.2. Possible global PROPerties.

(a) For each $a \in \mathcal{P}$ there is a transposition $t_{a} \in \operatorname{Aut}_{\mathbb{F}}(A)$ with

(i) $t_{a}^{2}=1 \neq t_{a}$ always;

(ii) $a^{t_{a}}=a$ and $\ell_{a, b}^{t_{a}}=\ell_{a, b} \quad$ always.

(b) On $A$ there is a nonzero bilinear form that is invariant in that $\langle\langle i j, k\rangle\rangle=$ $\langle\langle i, j k\rangle\rangle$ for all $i, j, k \in A$. 
Invariant forms have also been called associative forms and Frobenius forms [22, 23].

The transposition property is natural in our discussion. When 2.2 (a) holds, we extend the terminology introduced by Norton (for the case of 3-transposition groups) by calling an LN algebra admitting such automorphisms a transposition algebra. The dihedral group $\left\langle t_{a}, t_{b}\right\rangle$ acts naturally on the algebra $\ell_{a, b}$, so these line-algebras are often called dihedral-algebras.

Both Norton's algebra ${ }^{3} \| N^{\natural}$ and the Monster algebra $M^{\natural}$ are transposition algebras admitting nondegenerate invariant forms.

The existence of an invariant form is not only interesting but very helpful.

Lemma 2.3. Let $B$ be a commutative $\mathbb{F}$-algebra that admits the invariant bilinear from $\langle\langle\cdot, \cdot\rangle\rangle: B \times B \longrightarrow \mathbb{F}$. Then the radical

$$
\operatorname{Rad}(B)=\{x \in B \mid\langle\langle x, b\rangle\rangle=0 \text { for all } b \in B\}
$$

is an ideal of $B$.

Proof. The radical $R=\operatorname{Rad}(B)$ is clearly a subalgebra. As the form is invariant, for $r$ in the radical and $x, v$ arbitrary in $B$ we have

$$
\langle\langle v, x r\rangle\rangle=\langle\langle v x, r\rangle\rangle=0
$$

Therefore, for all $x \in B$ and $r \in R$ we also have $x r=r x \in R$; the radical $R$ is an ideal.

This often allows us to reduce to the study of simple algebras $A$ admitting forms with $\operatorname{Rad}(A)=0$; such forms are called nondegenerate.

\section{Geometric Presentations of Lie Algebras I}

We start with an example in which both desired LN global properties 2.2 for an algebra are consequences of its local definition. The construction is due to Kaplansky [31], although a version was apparently known to him and

\footnotetext{
${ }^{3}$ thought of as an algebra of dimension $2 \times 783$ over the reals
} 
others much earlier, as evidenced by papers by Block [1] and Kaplansky's student Hamelink [26].

Let $V$ be an $\mathbb{F}_{2}$-vector space admitting the symplectic form $(\cdot, \cdot)$ (that is, a bilinear form with $(x, x)=0$ always). Let $\mathcal{P}$ be a subset of the nonradical vectors in $V$ with the following property:

$$
\text { if } a, b \in \mathcal{P} \text { with }(a, b)=1 \text {, then } a+b \in \mathcal{P} \text {. }
$$

Theorem 3.1 (Kaplansky). Define on $A=\bigoplus_{a \in \mathcal{P}} \mathbb{F}_{2} v_{a}$ the LN algebra with presentation

$$
v_{a}^{2}=0 \quad \text { and } \quad v_{a} v_{b}=(a, b) v_{a+b}
$$

for all $a, b \in \mathcal{P}$. Then $A$ is a Lie algebra and $\left\langle\left\langle v_{a}, v_{b}\right\rangle\right\rangle=(a, b)$ extends to an invariant symplectic form on $A$.

Here $\epsilon=0$ and the set $\mathcal{A}$ of size three contains algebras $\ell_{a, b}$ of dimensions 1,2 , and 3 for the respective cases $a=b, a \neq b$ with $(a, b)=0$, and $a \neq b$ with $(a, b)=1$.

The elementary but crucial observation is that the restriction regarding 2-generated subalgebras actually determines the possibilities for 3-generated subalgebras. The only nontrivial case is the critical:

Lemma 3.2. If linearly independent $a, b, c \in \mathcal{P}$ with $(a, b)=1=(b, c)$ then $\mathbb{F}_{1} a+\mathbb{F}_{2} b+\mathbb{F}_{2} c=S$ is a symplectic 3 -subspace $\operatorname{Sp}_{3}(2)$ of $V$; that is, $S$ has radical of dimension 1 . Furthermore $S \backslash \operatorname{Rad}(S)=S \cap \mathcal{P}$.

This has two direct consequences:

(i) The Jacobi identity and the definition of form invariance (as in 2.2(b)) are linear in each of their three variables. Thus for the theorem they only need to be checked on the spanning set $\left\{v_{a} \mid a \in \mathcal{P}\right\}$, where the lemma can be applied.

(ii) The configuration $\mathcal{P}$ is left invariant by the group generated by the symplectic transvections centered at members of $\mathcal{P}$. Thus the LN algebra $A$ is in fact a transposition algebra with symplectic $\mathbb{F}_{2}$-transvections playing the role of transpositions. 
Kaplansky [31] used the theorem to construct three infinite families of simple Lie algebras over $\mathbb{F}_{2}$ - one already found by Block [1] and two others that appeared to be new (although one of those was essentially in Hamelink's thesis [26]).

The second consequence above - the existence of transposition automorphisms - allows the application of early results of McLaughlin [39] both to explain Kaplansky's simple examples and to suggest a path to classification.

Theorem 3.3 (McLaughlin [39]). There are exactly three types of finite dimensional irreducible groups generated by symplectic $\mathbb{F}_{2}$-transvections:

$$
\operatorname{Sym}(n), \mathrm{O}_{2 m}^{\epsilon}(2), \operatorname{Sp}_{2 m}(2) \text {. }
$$

\section{Transposition Groups and Algebras}

We focus on those groups generated by conjugacy classes of $k$-transpositions, particularly Fischer's 3-transpositions.

Definition 4.1 (Fischer [11]). Let $D$ be a conjugacy class of elements of order 2 in the group $G=\langle D\rangle$. Further assume that, for all $d, e \in D$, we have $|d e| \leq 3$. Then $(G, D)$ is called a 3 -transposition group and $D$ is its class of 3 -transpositions.

We may call $G$ a 3-transposition group when the class $D$ is evident. There is an obvious extension to normal sets $D$, but the most important case is that of a conjugacy class.

The case $|d e|=3$ occurs when the two involutions $d$ and $e$ generate a dihedral group of order 6 , a group that has many lives:

$$
\operatorname{Dih}_{6}=\operatorname{Sym}(3)=\mathrm{W}\left(A_{2}\right)=\operatorname{Sp}_{2}(2)=\mathrm{O}_{2}^{-}(2)=\mathrm{O}_{2}^{\text {Rad }}(3)=\mathrm{SU}_{2}(2) \text {. }
$$

Fischer's motivating example was the class of transpositions (that is, 2cycles) in a symmetric group, hence the name. But there are other examples as well. Specifically, the symplectic $\mathbb{F}_{2}$-transvections of the previous section are 3 -transpositions; so the symmetric groups are joined by the other groups from McLaughlin's Theorem 3.3, the orthogonal and symplectic groups over 
$\mathbb{F}_{2}$. Our list of aliases suggests the two further infinite classes of nearly simple 3-transposition groups - orthogonal groups over $\mathbb{F}_{3}$ and their reflections; unitary groups over $\mathbb{F}_{4}$ and their transvections.

Theorem 4.2 (Fischer [11]). A finite 3-transposition group with no nontrivial solvable normal subgroup is symmetric; symplectic or orthogonal over $\mathbb{F}_{2}$; orthogonal over $\mathbb{F}_{3}$; unitary over $\mathbb{F}_{4}$; or is isomorphic to one of the five groups

$$
\mathrm{P} \Omega_{8}^{+}(2) \rtimes \operatorname{Sym}(3), \mathrm{P} \Omega_{8}^{+}(3) \rtimes \operatorname{Sym}(3), F i_{22}, F i_{23}, F i_{24} .
$$

The two triality groups have orthogonal 3-transposition groups of index 3 , but the three other groups found by Fischer give rise to sporadic simple groups - the first two are simple while the third has the simple index 2 subgroup $F i_{24}^{\prime}$ (which we have mentioned earlier).

Fischer's classification was extended by Cuypers and Hall [10], who removed the finiteness condition and reduced the restriction on normal subgroups to requiring the center of $G$ to be trivial. There are many additional examples, but all are essentially variations on those found by Fischer.

The diagram of a set of 3-transpositions is the graph with that set as vertex set, two connected by an edge precisely when their product has order 3. This has importance because the 3-transposition group generated by that set is a quotient of the Coxeter group with the same simply-laced diagram. We have:

(i) Two elements of a normal set $S$ of 3-transpositions are conjugate in the group $G$ generated by the set precisely when they are in the same connected components of the diagram of $S$, and $G$ is a central product of 3-transposition groups associated with those components, since the corresponding results are already true for Coxeter groups.

(ii) The Weyl groups with simply-laced spherical Coxeter diagram $A_{n}, D_{n}$, $E_{n}$ are 3 -transposition groups with their generating reflections contained in the transposition class. The corresponding affine Weyl groups $\mathrm{W}(\tilde{X})$ for $X \in\left\{A_{n}, D_{n}, E_{n}\right\}$ are not, but their quotients $\mathrm{W}_{2}(\tilde{X})$ and $\mathrm{W}_{3}(\tilde{X})$ by, respectively, twice the root lattice and three times the root lattice are. For instance, $\mathrm{W}\left(A_{2}\right)=\operatorname{Sym}(3)$ (as noted above) and $\mathrm{W}\left(A_{3}\right)=\mathrm{W}_{2}\left(\tilde{A}_{2}\right)=\operatorname{Sym}(4)$. Also $\mathrm{W}_{3}\left(\tilde{A}_{2}\right)=\mathrm{SU}_{3}(2)^{\prime}$ of order 54 . 
(iii) Especially, any set of three 3-transpositions has diagram that is either spherical or affine, and so the group it generates is effectively known.

There is a natural generalization of Fischer's definition.

Definition 4.3. Let $D$ be a conjugacy class of elements of order 2 in the group $G=\langle D\rangle$. Further assume that, for all $d, e \in D$, we have $|d e| \leq$ $k$. Then $(G, D)$ is called a $k$-transposition group and $D$ is its class of $k$ transpositions.

This is relevant for us because the Monster $\mathbb{M}$ is a 6-transposition group [7]. For the conjugacy class of $2 A$ involutions in $\mathbb{M}$ there are nine conjugacy classes of 2-generated subgroups $\langle d, e\rangle$. These are dihedral subgroups of order $2 k$ with $k \leq 6$. The conjugacy class of the product $d e$ is then named $k X$ for $X \in\{A, B, C\}$, forming an extended $E_{8}$ picture (attributed to McKay []]):

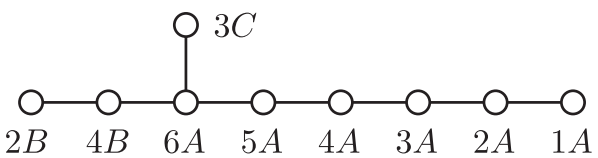

The Monster algebras of dimension 196884 arise (at least as modules) from the rank 9 conjugation action of $\mathbb{M}$ on its $2 A$ class. Conway observed that the 1-space of $M^{\natural}$ fixed by the centralizer of a $2 A$ involution contains an idempotent, which he called an axis. The involution acts on $M^{\natural}$ as the corresponding Miyamoto involution. This reveals $M^{\natural}$ as a transposition algebra where the isomorphism classes for subalgebras generated by two axes form a set $\mathcal{A}$ of size 9 . If the $2 A$ involutions $d$ and $e$ have product in class $k X$ (as given above), then the corresponding line/dihedral-algebra $\ell_{d, e}$ is named $k X$.

These nine 2-generated subalgebras of $M^{\natural}$ are described in detail by Conway and Norton [7]. They naturally arise in other related situations. Especially Sakuma [47] proved that in a compact Griess algebra (in the sense of Miyamoto) any subalgebra generated by two Ising vectors (conformal vectors of central charge $\frac{1}{2}$ ) is isomorphic to one of these nine. The same classification occurs in the more general contexts of [29] and [22]; see Section 7 for further discussion. 
Example 4.4 (Certain Conway-Norton-Sakuma algebras). Although $M^{\natural}$ is an algebra over the reals, it makes sense to define these algebras over arbitrary fields $\mathbb{F}$.

(i) The algebra $1 A$ is generated by the single axis $z_{0}=1$ and is $\mathbb{F}$.

(ii) The algebra $2 B$ is the 2-dimensional associative algebra $\mathbb{F} \oplus \mathbb{F}$ with $\left\{b_{0}, b_{1}\right\}$ a basis of axes having the relations

$$
b_{0}^{2}=b_{0}, b_{1}^{2}=b_{1}, b_{0} b_{1}=0
$$

(iii) Assume $\operatorname{char}(\mathbb{F}) \neq 2$. The algebra $2 A$ is a 3 -dimensional algebra with $\left\{a_{0}, a_{1}, a_{2}\right\}$ a basis of axes subject (for $\{i, j, k\}=\{0,1,2\}$ ) to the relations

$$
a_{i}^{2}=a_{i}, a_{i} a_{j}=\frac{1}{8}\left(a_{i}+a_{j}-a_{k}\right) .
$$

(iv) Assume $\operatorname{char}(\mathbb{F}) \neq 2$. The algebra $3 C$ is a 3 -dimensional algebra with $\left\{c_{0}, c_{1}, c_{2}\right\}$ a basis of axes subject (for $\{i, j, k\}=\{0,1,2\}$ ) to the relations

$$
c_{i}^{2}=c_{i}, c_{i} c_{j}=\frac{1}{64}\left(c_{i}+c_{j}-c_{k}\right)
$$

\section{Point-line Geometry}

\subsection{Definitions and examples}

We have used the terminology of points and lines in the context of LN algebras because the associated presentations are often described via a geometric "skeleton" of points and lines. For instance, Kaplansky's algebras of Theorem 3.1 were defined via a set of projective points and lines embedded in a symplectic geometry over $\mathbb{F}_{2}$.

\section{Definition 5.1.}

(i) A point-line geometry $(\mathcal{P}, \mathcal{L})$ is a point set $\mathcal{P}$ and a line set $\mathcal{L}$ such that each line is a subset of $\mathcal{P}$ of size at least 2.

(ii) A point-line geometry is a partial linear space if every pair of points is in at most one line and a linear space if every pair of points is in a unique line. 
(iii) A partial linear space $(\mathcal{P}, \mathcal{L})$ is a partial triple system if every line is a 3 -subset of $\mathcal{P}$; it is a Steiner triple system if it is additionally a linear space.

(iv) A point-line geometry is connected if it is possible to "walk along" lines from any point to any other. That is, the collinearity graph on its point set is connected.

(v) A subspace $\left(\mathcal{P}^{\prime}, \mathcal{L}^{\prime}\right)$ of a point-line geometry $(\mathcal{P}, \mathcal{L})$ is a subset $\mathcal{P}^{\prime}$ of $\mathcal{P}$ that contains all points of every line of $\mathcal{L}$ meeting it in at least two points, equipped with the line set $\mathcal{L}^{\prime}$ of all such lines.

(vi) A plane of a point-line geometry is a subspace generated by two intersecting lines - the intersection of all subspaces containing the two lines and the points on them.

\section{Example 5.2.}

(i) The points and lines of any projective space form a linear space. Especially when the field is $\mathbb{F}_{2}$, the points and lines form the Steiner triple system $\mathrm{PG}_{n}(2)$ (where $n$ is the rank, one less than the vector space dimension).

(ii) Similarly the points and lines of any affine space form a linear space, and over the field $\mathbb{F}_{3}$ we have the Steiner triple system $\operatorname{AG}_{n}(3)$, where $n$ is the vector space dimension.

(iii) For Kaplansky's algebras from Section 3 we are considering subspaces of a symplectic geometry $\operatorname{Sp}_{n}(2)$, where the points are nonradical vectors (1-spaces) and the lines are the 2-spaces that are nondegenerate under restriction of the form.

(iv) All planes of $\mathrm{PG}_{n}(2)$ are $\mathrm{PG}_{2}(2)$.

(v) All planes of $\mathrm{AG}_{n}(3)$ are $\mathrm{AG}_{2}(3)$. Equally well this is $\mathrm{O}_{3}^{+}(3)$ : the 1spaces of square length in a nondegenerate orthogonal 3-space over $\mathbb{F}_{3}$ with lines the 2-spaces $\mathrm{O}_{2}^{\mathrm{Rad}}(3)$ whose radical has dimension 1 . It is also $\mathrm{SU}_{3}(2)$ : the singular 1-spaces in a nondegenerate unitary 3 -space over $\mathbb{F}_{4}$ with lines the nondegenerate 2 -spaces.

(vi) All planes of $\operatorname{Sp}_{n}(2)$ are $\operatorname{Sp}_{3}(2)=\mathrm{AG}_{2}(2)^{*}$, the symplectic space of dimension 3 with radical of dimension 1 . Equally well this is the dual affine plane of order 2 : the projective $\mathrm{PG}_{2}(2)$ with a point and all lines through it deleted. $\mathrm{Sp}_{3}(2)$ is also called the Pasch configuration. 


\subsection{Fischer spaces}

We have seen above that the planes $\operatorname{Sp}_{3}(2)$ and $\mathrm{AG}_{2}(3)$ lead multiple lives. For us, the most important subclass of point-line geometries will be:

Definition 5.3 (Buekenhout [4]). The connected partial triple system $(\mathcal{P}, \mathcal{L})$ is a Fischer space provided its planes are $\mathrm{Sp}_{3}(2)$ or $\mathrm{AG}_{2}(3)$. It is said to be of symplectic type if only $\mathrm{Sp}_{3}(2)$ occurs.

Theorem 5.4 (Buekenhout [4]). Fischer spaces and 3-transposition groups "are the same thing."

Formally, appropriate categories of Fischer spaces and 3-transposition groups are equivalent. Thus Fischer spaces were classified by Fischer [11] and Cuypers and Hall [10]. Those of symplectic type were identified by Shult [49] and Hall [19, 20].

We sketch a proof of the theorem:

(a) Let $(\mathcal{P}, \mathcal{L})$ be a Fischer space. For each $p \in \mathcal{P}$ define $t_{p} \in$ $\operatorname{Sym}(\mathcal{P})$ to be the involution that fixes $p$ and each $q$ not collinear with $p$ and switches $q$ and $r$ when $\{p, q, r\} \in \mathcal{L}$. Then $D(\mathcal{P}, \mathcal{L})=\left\{t_{p} \mid p \in \mathcal{P}\right\}$ is a conjugacy class of 3transpositions in the subgroup $\langle D(\mathcal{P}, \mathcal{L})\rangle=G(\mathcal{P}, \mathcal{L})$ of the automorphism group $\operatorname{Aut}(\mathcal{P}, \mathcal{L})$.

(b) Let the 3-transposition group $G$ be generated by the class $D$ of 3-transpositions. Set $\mathcal{P}(D)=D$, and let $\mathcal{L}(D)$ consist of the triples $D \cap S$ as $S$ runs through all $D$-generated subgroups isomorphic to $\operatorname{Sym}(3)$. Then $(\mathcal{P}(D), \mathcal{L}(D))$ is a Fischer space.

(c) If we start with a Fischer space $(\mathcal{P}, \mathcal{L})$, move to the 3 -transposition group $G(\mathcal{P}, \mathcal{L})$ as in (a) and then back as in (b), the resulting Fischer space

$$
(\mathcal{P}(D(\mathcal{P}, \mathcal{L})), \mathcal{L}(D(\mathcal{P}, \mathcal{L})))
$$

is isomorphic to $(\mathcal{P}, \mathcal{L})$.

The first part can be checked within subspaces generated by a point and a line of $(\mathcal{P}, \mathcal{L})$; it is immediate except in planes where it follows directly. The second can be checked in 3-generated subgroups; each 3-subset of $D$ has 
a diagram that is either spherical or affine as Coxeter diagram. The third part then follows. Conjugacy corresponds to connectivity. See [19, (3.2)] for details of these arguments. 4 $^{4}$

\subsection{Triple and Matsuo algebras}

Let $(\mathcal{P}, \mathcal{L})$ be a partial triple system. An associated triple algebra $\mathrm{A}(\mathcal{P}, \mathcal{L})$ is an LN algebra $A=\bigoplus_{p \in \mathcal{P}} \mathbb{F} p$ with presentation

(i) $i^{2}=\epsilon i$ for all $i \in \mathcal{P}$;

(ii) $i j=0$ if $i \neq j$ but there is no $k$ with $\{i, j, k\} \in \mathcal{L}$;

(iii) $i j=\alpha i+\beta j+\gamma k$ when $\{i, j, k\} \in \mathcal{L}$,

where $\alpha, \beta, \gamma$, and $\epsilon$ are constants from $\mathbb{F}$.

In an algebra $\mathrm{A}(\mathcal{P}, \mathcal{L})$ associated with $(\mathcal{P}, \mathcal{L})$, each line-algebra $\ell_{a, b}$ is determined by the line $l \in \mathcal{L}$ on the points $a, b \in \mathcal{P}$. Especially $a b=0$ if $a, b$ are not collinear in $\mathcal{L}$, so in that case $\ell_{a, b}$ is a copy of the $2 B$ algebra $\mathbb{F} \oplus \mathbb{F}$.

If the partial triple system $(\mathcal{P}, \mathcal{L})$ is disconnected, being the disjoint union of two subspaces $\left(\mathcal{P}_{1}, \mathcal{L}_{1}\right)$ and $\left(\mathcal{P}_{2}, \mathcal{L}_{2}\right)$ with no point of $\mathcal{P}_{1}$ collinear with any point of $\mathcal{P}_{2}$, then $\mathrm{A}(\mathcal{P}, \mathcal{L})$ is the algebra direct sum of the two algebras $\mathrm{A}\left(\mathcal{P}_{1}, \mathcal{L}_{1}\right)$ and $\mathrm{A}\left(\mathcal{P}_{2}, \mathcal{L}_{2}\right)$. This allows us to focus on connected partial triple systems $(\mathcal{P}, \mathcal{L})$ when desirable.

Algebras $\mathrm{A}(\mathcal{P}, \mathcal{L})$ for Fischer spaces $(\mathcal{P}, \mathcal{L})$ will appear in the next section. Of related importance is the Matsuo algebra associated with a partial triple system $(\mathcal{P}, \mathcal{L})$. It is the triple algebra $\mathrm{A}(\mathcal{P}, \mathcal{L})=\bigoplus_{p \in \mathcal{P}} \mathbb{F} p$ given by

$$
i^{2}=i \quad \text { and } \quad i j=\alpha(i+j-k) \text { for }\{i, j, k\} \in \mathcal{L},
$$

\footnotetext{
${ }^{4}$ The correspondence just discussed is not quite the category equivalence claimed above. For $(G, D)$ an arbitrary 3-transposition group, after the double transition

$$
G \stackrel{(\mathrm{b})}{\longrightarrow}(\mathcal{P}(D), \mathcal{L}(D)) \stackrel{(\mathrm{a})}{\longrightarrow} G(\mathcal{P}(D), \mathcal{L}(D))
$$

we finish with $G(\mathcal{P}(D), \mathcal{L}(D))$ isomorphic to $G / \mathrm{Z}(G)$, not always isomorphic to $G$. To remedy this and other problems, we must replace each 3-transposition group by an appropriate "universal central extension" and consider the subcategory of such universal groups. This is similar to the situation encountered in [21].
} 
so that $\alpha=\beta=-\gamma$ and $\epsilon=1$. These were introduced by Matsuo and Matsuo [38, 36, 37].

In Matsuo algebras each point yields an idempotent generating a $1 A$ algebra $\mathbb{F}$.

For characteristic other than 2, the Matsuo line-algebras $\ell_{i, j}=\ell_{j, k}=\ell_{i, k}$ where $\{i, j, k\} \in \mathcal{L}$ are said to have type $3 C(\eta)$ for $\eta=2 \alpha$, after [23]. Certain of these are familiar (and motivate the notation).

\section{Lemma 5.5.}

(a) The algebra $3 C$ is the Matsuo algebra $3 C\left(\frac{1}{32}\right)$.

(b) The algebra $2 A$ is the Matsuo algebra $3 C\left(\frac{1}{4}\right)$.

(c) The Matsuo algebra $3 C\left(\frac{1}{2}\right)$ is a Jordan algebra.

As we shall discuss in Section 8 , the case $\eta=\frac{1}{2}$ is quite exceptional.

\section{Geometric Presentations of Lie Algebras II}

\subsection{Kaplansky's algebras}

For the partial triple system $(\mathcal{P}, \mathcal{L})$, the triple algebra of Kaplansky type $\mathrm{A}(\mathcal{P}, \mathcal{L})=\bigoplus_{p \in \mathcal{P}} \mathbb{F}_{2} p$ is given by

$$
i^{2}=0 \quad \text { and } \quad i j=k \quad \text { for }\{i, j, k\} \in \mathcal{L}
$$

When $(\mathcal{P}, \mathcal{L})$ is a subspace of one of the symplectic partial triple systems $\mathrm{Sp}_{n}(2)$, this is precisely one of Kaplansky's algebras. These algebras have been regularly rediscovered and studied. The definitive results are due to Cuypers.

Theorem 6.1 (Cuypers [8]). Assume $(\mathcal{P}, \mathcal{L})$ is connected. The algebra $\mathrm{A}(\mathcal{P}, \mathcal{L})$ above is a Lie algebra if and only if $(\mathcal{P}, \mathcal{L})$ is a Fischer space of symplectic type.

(i) This explains Kaplansky's construction and gives a classification, since Fischer spaces of symplectic type are classified.

(ii) McLaughlin's symplectic transvections are recognized as examples of symplectic 3 -transpositions (hence the terminology). 
The algebras defined above are clearly commutative, so they can only hope to be Lie algebras in characteristic 2. A slight variation gives access to Lie algebras over arbitrary fields:

Theorem 6.2 (Brouwer, Cohen, Cuypers, Hall, Postma [3]). Given a connected partial triple system $(\mathcal{P}, \mathcal{L})$, consider a point-line algebra $\mathrm{B}(\mathcal{P}, \mathcal{L})=$ $\bigoplus_{p \in \mathcal{P}} \mathbb{F} p$ given by

(i) $i^{2}=0$ for all $i \in \mathcal{P}$;

(ii) $i j=0$ if $i \neq j$ but there is no $k$ with $\{i, j, k\} \in \mathcal{L}$;

(iii) $i j= \pm k$ when $\{i, j, k\} \in \mathcal{L}$.

Then the signs can be chosen so that $\mathrm{B}(\mathcal{P}, \mathcal{L})$ is a Lie algebra if and only if we have one of:

(a) $(\mathcal{P}, \mathcal{L})$ is a Fischer space of symplectic type;

(b) $\mathbb{F}$ has characteristic 3 and $(\mathcal{P}, \mathcal{L})$ consists of the points and lines disjoint from a fixed subspace $\mathrm{PG}_{m}(2)$ of a projective space $\mathrm{PG}_{m+2}(2)$.

In all successful cases $\mathrm{B}(\mathcal{P}, \mathcal{L})$ is uniquely determined up to isomorphism.

The new examples are associated with nonsimplicity of the Lie algebra $\mathfrak{g}_{2}$ in characteristic 3 .

\subsection{More Lie and non-Lie algebras from Fischer spaces}

For $(\mathcal{P}, \mathcal{L})$ a Fischer space, consider the triple algebra $\mathrm{A}(\mathcal{P}, \mathcal{L})=\bigoplus_{p \in \mathcal{P}} \mathbb{F}_{2} p$ with

$$
i^{2}=0 \quad \text { and } \quad i j=i+j+k \quad \text { for }\{i, j, k\} \in \mathcal{L}
$$

so that $\epsilon=0$ and $\alpha=\beta=\gamma=1$. This algebra admits a natural invariant symplectic form on $\mathcal{P}$ given by:

$$
\begin{array}{rr}
\langle\langle i, j\rangle\rangle=1 & \text { if } i, j \text { are distinct and collinear; } \\
\langle\langle i, j\rangle\rangle=0 & \text { otherwise. }
\end{array}
$$

Set $\overline{\mathrm{A}}(\mathcal{P}, \mathcal{L})=\mathrm{A}(\mathcal{P}, \mathcal{L}) / \operatorname{Rad}(\mathrm{A}(\mathcal{P}, \mathcal{L}))$, still an algebra by Lemma 2.3, 
Cuypers, Horn, in 't panhuis, and Shpectorov [9] studied these algebras with an eye open for Lie algebras. They found a characterization in the same spirit as Cuypers' Theorem 6.1.

Theorem 6.3 (Cuypers, Horn, in 't panhuis, Shpectorov [9]). The algebra $\overline{\mathrm{A}}(\mathcal{P}, \mathcal{L})$ is a Lie algebra if and only if the Fischer space $(\mathcal{P}, \mathcal{L})$ contains no subspace $\mathrm{AG}_{3}(3)$, an affine 3 -space over $\mathbb{F}_{3}$.

They next listed the appropriate Fischer spaces and examined the associated Lie algebras.

Example 6.4 (NICE Lie ExAmPles [9]). We have $\mathrm{P} \Omega_{8}^{+}(2): 2 \leq \mathrm{P} \Omega_{8}^{+}(2) \rtimes \operatorname{Sym}(3) \leq F i_{22}$. The associated chain of quotient algebras consists of Lie algebras.

(a) $\operatorname{dim}_{\mathbb{F}_{2}} \bar{A}\left(\mathrm{P} \Omega_{8}^{+}(2): 2\right)=8$, an abelian Lie algebra;

(b) $\operatorname{dim}_{\mathbb{F}_{2}} \bar{A}\left(\mathrm{P} \Omega_{8}^{+}(2) \rtimes \operatorname{Sym}(3)\right)=26(=2+3 \times 8)$, a Lie algebra of type $D_{4}(2)$;

(c) $\operatorname{dim}_{\mathbb{F}_{2}}\left(\bar{A}\left(F i_{22}\right)\right)=78$, a Lie algebra of type ${ }^{2} E_{6}(2)$.

Fischer had earlier shown $F i_{22} \leq{ }^{2} E_{6}(2)$, and here we see that observation in a broader context.

Cuypers, Horn, in 't panhuis, and Shpectorov went on to calculate many examples that were not Lie algebras.

Example 6.5 (NiCE Non-Lie Examples [9]). We have $\mathrm{P} \Omega_{8}^{+}(3): 2 \leq \mathrm{P} \Omega_{8}^{+}(3) \rtimes \operatorname{Sym}(3) \leq F i_{23}$. The associated chain of quotient algebras contains no Lie algebras.

(a) $\operatorname{dim}_{\mathbb{F}_{2}} \bar{A}\left(\mathrm{P} \Omega_{8}^{+}(3): 2\right)=260$;

(b) $\operatorname{dim}_{\mathbb{F}_{2}} \bar{A}\left(\mathrm{P} \Omega_{8}^{+}(3) \rtimes \operatorname{Sym}(3)\right)=782(=2+3 \times 260)$;

(c) $\bar{A}\left(F i_{23}\right)=\bar{A}\left(\mathrm{P} \Omega_{8}^{+}(3) \rtimes \operatorname{Sym}(3)\right)$ of dimension 782 .

This suggests an elegant construction of the sporadic 3-transposition group $F i_{23}$ as the automorphism group of a quotient triple algebra of dimension 782 arising naturally from the triality group $\mathrm{P} \Omega_{8}^{+}(3) \rtimes \operatorname{Sym}(3)$.

Our starting point was Simon Norton's construction of $3 \cdot F i_{24}^{\prime}$ as automorphisms of a triple algebra of complex dimension 783. These last examples 
suggest an elegant $\mathbb{F}_{4}$-triple algebra version of the original Norton algebra in dimension 783.

\section{Axial Algebras with Ising Fusion}

We begin with another algebra definition which, by itself, is too general. An idempotent $e$ in the $\mathbb{F}$-algebra $A$ is an axis if its associated adjoint linear operator

$$
\operatorname{ad}_{e}: A \longrightarrow A \text { given by } a \mapsto a e
$$

is semisimple. That is, if the minimal polynomial of $\operatorname{ad}_{e}$ is square-free. For associative algebras, the minimal polynomial divides $x^{2}-x$ and always splits. For nonassociative algebras there may be additional eigenvalues and the adjoint need not be semisimple. It is a well-known theorem [30, II.11] that in Jordan algebras all idempotents are ad-semisimple but with eigenvalues from $\left\{1,0, \frac{1}{2}\right\}$.

An axial algebra $(A, \mathcal{A})$ is then a commutative algebra $A$ generated by a set of axes $\mathcal{A}$. (Mention of the generating set $\mathcal{A}$ is sometimes omitted.) Any Jordan algebra generated by idempotents gives an example. An axis is primitive if it spans its own 1-eigenspace. 5 Similarly, an axial algebra $(A, \mathcal{A})$ is primitive if it is generated by the set $\mathcal{A}$ of primitive axes.

We narrow the field by restricting the fusion properties of the idempotents. For the subset $\Lambda \subseteq \mathbb{F}$, a fusion rule is a map $\mathcal{F}: \Lambda \times \Lambda \longrightarrow 2^{\Lambda}$. The decomposition $A=\bigoplus_{\lambda \in \Lambda} A_{\lambda}$ obeys the fusion rule if, for all $\lambda, \mu \in \Lambda$ we have

$$
A_{\lambda} A_{\mu} \subseteq \sum_{\nu \in \mathcal{F}(\lambda, \mu)} A_{\nu}
$$

An axis $e$ in $A$ is an $\mathcal{F}$-axis if $\Lambda$ is a subset containing the eigenvalues of $e$ and the eigenspace decomposition for $\operatorname{ad}_{e}$

$$
A=\bigoplus_{\lambda \in \Lambda} A_{\lambda}(e)
$$

\footnotetext{
${ }^{5}$ This is a stricter definition of primitivity than is standard for Jordan algebras [30, p. 158].
} 
obeys $\mathcal{F}$ (where $A_{\lambda}(e)$ is the $\lambda$-eigenspace for $\operatorname{ad}_{e}$ ). An axial algebra $(A, \mathcal{A})$ is an $\mathcal{F}$-axial algebra if it is generated by the set $\mathcal{A}$ of $\mathcal{F}$-axes.

If we set $\mathcal{F}(\lambda, \mu)=\mathbb{F}$ for all pairs $\lambda, \mu$ then every idempotent is an $\mathcal{F}$ axis, and we have learned nothing. The cases of interest are those with each $\mathcal{F}(\lambda, \mu)$ small. For instance, if always $|\mathcal{F}(\lambda, \mu)| \leq 1$ then we have a grading by a magma within $\Lambda \cup\{\emptyset\}$ whose multiplication satisfies $\lambda \star \mu \subseteq \mathcal{F}(\lambda, \mu)$.

The original fusion rule of interest to us is Ising fusion $\mathcal{I}$ given by the fusion table

\begin{tabular}{|c|c|c|c|c|c|}
\hline & $\mathcal{I}$ & 1 & + & $\frac{1}{4}$ & $\begin{array}{l}- \\
\frac{1}{32} \\
\end{array}$ \\
\hline \multirow{3}{*}{+} & 1 & 1 & $\emptyset$ & $\frac{1}{4}$ & $\frac{1}{32}$ \\
\hline & 0 & $\emptyset$ & 0 & $\frac{1}{4}$ & $\frac{1}{32}$ \\
\hline & $\frac{1}{4}$ & $\frac{1}{4}$ & $\frac{1}{4}$ & 1,0 & $\frac{1}{32}$ \\
\hline- & $\frac{1}{32}$ & $\frac{1}{32}$ & $\frac{1}{32}$ & $\frac{1}{32}$ & $1,0, \frac{1}{4}$ \\
\hline
\end{tabular}

Conway [7] observed that in $M^{\natural}$ there are 1-dimensional subalgebras generated by ad-semisimple idempotents for $\Lambda=\left\{1,0, \frac{1}{4}, \frac{1}{32}\right\}$ (after rescaling) and called such an element an axis. Miyamoto [40] noted that, with respect to the embedding of $M^{\natural}$ as $V_{2}^{\natural}$ within the Monster vertex operator algebra $V^{\natural}$, these axes are spanned by conformal vectors of central charge $\frac{1}{2}$. Such vectors give rise to vertex operator action by the Virasoro algebra of central charge $\frac{1}{2}$. The representation theory of this Lie algebra is well-understood, and Miyamoto used it to prove that the associated idempotents are primitive $\mathcal{I}$-axes. Indeed, Miyamoto proved this for the conformal vectors of central charge $\frac{1}{2}$ in any compact Griess algebra $V_{2}$. (The vectors are sometimes called Ising vectors [47].)

As noted by Miyamoto, a striking consequence of this is that associated to each Ising idempotent $e$ there is an automorphism $t_{e}$ of the Griess algebra $A=V_{2}$ (and indeed of $V$ itself). This is a simple consequence of the Ising fusion rule. As suggested in the fusion table, we set $\Lambda_{+}=\left\{1,0, \frac{1}{4}\right\}$ and 
$\Lambda_{-}=\left\{\frac{1}{32}\right\}$. Then the decomposition $A=A_{+}(e) \oplus A_{-}(e)$ is a grading of the algebra $A$ by the group $\{ \pm 1\}=\{ \pm\}$ of order 2 :

$$
A_{\delta}(e) A_{\epsilon}(e) \subseteq A_{\delta \epsilon}(e) \quad \text { for } \quad \delta, \epsilon \in\{ \pm\} .
$$

Therefore

$$
t_{e}: A \longrightarrow A \text { given by } t_{e}: a_{+}+a_{-} \mapsto a_{+}-a_{-}
$$

is an automorphism of $A$. We have $t_{e}$ of order 1 or 2 depending upon whether $A_{\frac{1}{32}}(e)$ is trivial. In the nontrivial case Miyamoto called $t_{e}$ a $\tau$-involution (using notation $\tau_{e}$ ). These give the motivating examples of what we call Miyamoto involutions.

We have the crucial result:

Theorem 7.1 (Sakuma's Theorem [47]). Let A be the compact Griess algebra of an $\mathrm{OZ}$ vertex operator algebra. The subalgebra of A generated by two Ising vectors is isomorphic to one of the nine algebras

$$
1 A, 2 A, 3 A, 4 A, 5 A, 6 A, 2 B, 4 B, 3 C
$$

found by Conway and Norton [7] as subalgebras of $M^{\natural}$ generated by two axes.

The four important examples $1 A, 2 B, 2 A, 3 C$ were given in 4.4 .

Sakuma's Theorem reveals the axial algebra generated by the Ising vectors within a compact Griess algebra as a transposition algebra, the transpositions being provided by the $\tau$-involutions. . $^{-1}$

A compact Griess algebra has various properties in addition to being a primitive Ising axial algebra [40]. It is a real algebra $A$ equipped with a symmetric bilinear form $\langle\langle\cdot, \cdot\rangle\rangle$ that:

(i) is invariant;

(ii) is positive definite;

(iii) satisfies the Norton inequality: $\langle\langle a, a\rangle\rangle\langle\langle b, b\rangle\rangle \geq\langle\langle a b, a b\rangle\rangle$ for all $a, b \in A$.

\footnotetext{
${ }^{6}$ This assumes $\tau \neq 1$. See the next section for discussion of the case $\tau=1$.
} 
Ivanov [28, 8.6.1] stepped away from the arena of vertex operator algebras by defining a class of algebras that enjoy certain of the properties noted by Miyamoto. Ivanov's algebras are defined as real, primitive, Ising axial algebras equipped with a form satisfying the above properties (i)-(iii). Since the axes of Ivanov's algebras obey the Ising fusion rules, each axis admits the corresponding Miyamoto $(\tau$-)involution, called by Ivanov a Majorana involution.

Ivanov and others were able to duplicate many results on Griess algebras in this context. Especially Ivanov, Pasechnik, Seress, and Shpectorov [29] proved that Sakuma's Theorem remains valid.

Later Hall, Rehren, and Shpectorov [22] proved that Sakuma's Theorem holds for any primitive Ising axial algebra admitting a nontrivial invariant form.

\section{Axial Algebras of Jordan Type}

The results of this section are all from Hall, Rehren, and Shpectorov [23], one of the two papers (the other being [22]) where axial algebras were first defined and studied.

It must be noted that portions of the results in this section had been proven by Matsuo ten years earlier in [36], the unpublished original version of the published paper [37]. Matsuo's results were in the more restricted context of compact Griess algebras (allowing him to make use of a positive definite, invariant form), but it is regrettable that his results were unremarked for so long.

In 40] Miyamoto did not restrict his attention to the Ising case. He showed that each conformal vector (idempotent) in the Griess algebra of an $\mathrm{OZ}$ vertex operator algebra is associated with a vertex operator action by the Virasoro algebra with an appropriate central charge $c$. The representation theory of that Virasoro algebra then determines a fusion rule satisfied by the eigenspaces of the corresponding axis/conformal vector. If that representation theory is nice enough, then more can be done. For instance the fusion rule may dictate a grading and hence automorphisms similar to the Miyamoto $\tau$-involutions. The Ising situation is far better understood than any other, with the possible exception of fusion of Jordan type, which we discuss in this section. 
In the previous section we said that the Ising vector $e$ of a Griess algebra $A$ provides a transposition in the form of the $\tau$-involution of Miyamoto. This was loose, since there is the possibility that the resulting $t_{e}$ is trivial; that happens when $A_{\frac{1}{32}}(e)=0$. Miyamoto observed that in this case, there is nevertheless a good candidate transposition. When the $\frac{1}{32}$-eigenspace is trivial, the resulting reduced fusion table is

\begin{tabular}{c||cc|c}
$\mathcal{I}$ & 1 & 0 & $\frac{1}{4}$ \\
\hline \hline 1 & 1 & $\emptyset$ & $\frac{1}{4}$ \\
0 & $\emptyset$ & 0 & $\frac{1}{4}$ \\
\hline$\frac{1}{4}$ & $\frac{1}{4}$ & $\frac{1}{4}$ & 1,0
\end{tabular}

Here we have a different \pm -grading, namely

$$
A_{+}(e)=A_{1}(e) \oplus A_{0}(e) \quad \text { and } \quad A_{-}(e)=A_{\frac{1}{4}}(e)
$$

This new grading provides a new transposition automorphism $t_{e}$, called a $\sigma$-involution by Miyamoto.?

Compare this with the case of an Ising vector for which the $\frac{1}{4}$-eigenspace is trivial. There the reduced fusion table is

\begin{tabular}{c||cc|c}
$\mathcal{I}$ & 1 & 0 & $\frac{1}{32}$ \\
\hline \hline 1 & 1 & $\emptyset$ & $\frac{1}{32}$ \\
0 & $\emptyset$ & 0 & $\frac{1}{32}$ \\
\hline$\frac{1}{32}$ & $\frac{1}{32}$ & $\frac{1}{32}$ & 1,0
\end{tabular}

and the $\tau$-involution remains an algebra transposition.

In the rest of this section, the only assumption we make for the field $\mathbb{F}$ is that $\operatorname{char}(\mathbb{F}) \neq 2$.

\footnotetext{
${ }^{7}$ An Ising vector $e$ having $V_{\frac{1}{32}}(e)=0=V_{\frac{1}{4}}(e)$ associates with everything, with $\tau_{e}$ and then $\sigma_{e}$ both being trivial. We will not discuss this degenerate case further.
} 
Let $\eta \in \mathbb{F}$ be a constant not equal to 0 or 1 . The two fusion tables above are the special cases $\eta=\frac{1}{4}$ and $\eta=\frac{1}{32}$ of the fusion rule $\mathcal{J}_{\eta}$ of Jordan type $\eta$ :

\begin{tabular}{c||cc|c}
$\mathcal{J}_{\eta}$ & 1 & 0 & $\eta$ \\
\hline \hline 1 & 1 & $\emptyset$ & $\eta$ \\
0 & $\emptyset$ & 0 & $\eta$ \\
\hline$\eta$ & $\eta$ & $\eta$ & 1,0
\end{tabular}

The terminology arises from the fact that any idempotent of a Jordan algebra is an axis of Jordan type $\frac{1}{2}$. That is, its eigenspace decomposition obeys the Jordan fusion rule with $\eta=\frac{1}{2}$. This is a well-known property of the Peirce decomposition; see [30, p. 119].

For arbitrary $\eta$ we have the \pm -grading

$$
A_{+}(e)=A_{1}(e) \oplus A_{0}(e) \quad \text { and } \quad A_{-}(e)=A_{\eta}(e)
$$

so each axis admits a Miyamoto involution. Again, this is well-known for Jordan algebras, which fall under the case $\eta=\frac{1}{2}$.

A $\mathcal{J}_{\eta}$-axial algebra is said to have Jordan type $\eta$. Proposition 8.2 and Theorem 8.3 below give an effective classification of all primitive axial algebras of Jordan type $\eta \neq \frac{1}{2}$.

The case $\eta=\frac{1}{2}$ is genuinely exceptional, not just because it includes certain Jordan algebras. For instance, as noted by Segev, the result [23, $(5.4)]$ :

$$
t_{a}=t_{b} \Longleftrightarrow a=b,
$$

is true for $\eta \neq \frac{1}{2}$ but false for $\eta=\frac{1}{2}$, a situation discussed at length and resolved in [24]. 8 ]

\footnotetext{
${ }^{8}$ The falsity of [23, (5.4)] for $\eta=\frac{1}{2}$ does not affect any other results of that paper, but two proofs in which it was invoked must be modified. The corollary [23, (5.5)] remains valid, but its proof must be supported by [24, Theorem B] for the case $\eta=\frac{1}{2}$. The error also invalidated the proof of [23, (6.3)] (but not the result) in the case $\eta=\frac{1}{2}$. The paper [25] from the current volume provides, among other results, a proof of that result valid for all $\eta$. No other results of [23] appeal to the incorrect case $\eta=\frac{1}{2}$ of $[23$, (5.4)].
} 
Recall from Section 5.3 that the Matsuo algebra associated with the partial triple system $(\mathcal{P}, \mathcal{L})$ is the triple algebra $\mathrm{A}(\mathcal{P}, \mathcal{L})=\bigoplus_{p \in \mathcal{P}} \mathbb{F} p$ with presentation, for a constant $\alpha \in \mathbb{F}$ :

$$
\begin{array}{rlr}
1 A: & i^{2}=i & \text { for } i \in \mathcal{P} ; \\
3 C(2 \alpha): & i j=\alpha(i+j-k) & \text { for }\{i, j, k\} \in \mathcal{L} ; \\
2 B: & i j=0 & \text { otherwise. }
\end{array}
$$

Proposition 8.1. The Matsuo algebra $\mathrm{A}(\mathcal{P}, \mathcal{L})$ is a primitive axial algebra generated by the axes of $\mathcal{P}$, each with eigenvalues from $\{1,0, \eta\}$ where $\eta=2 \alpha$.

Proposition 8.2. Assume $(\mathcal{P}, \mathcal{L})$ is connected. Let $M=\mathrm{A}(\mathcal{P}, \mathcal{L})$ be a Matsuo algebra with $\eta \neq \frac{1}{2}$. Then the following are equivalent:

(1) $(\mathcal{P}, \mathcal{L})$ is a Fischer space.

(2) $M$ admits a transposition (Miyamoto involution) at each point of $\mathcal{P}$.

(3) $M$ is an axial algebra of Jordan type $\eta$.

In this case, $M$ admits a nontrivial invariant form that is unique up to a scalar multiple. Furthermore, the radical of that form is the unique maximal ideal containing no axes.

Theorem 8.3. Let $A$ be a primitive axial algebra of Jordan type $\eta \neq \frac{1}{2}$. Then the Miyamoto involutions for the associated axes form a normal set of 3-transpositions in the automorphism group $\operatorname{Aut}(A)$. Indeed $A$ is isomorphic to a quotient of the corresponding Matsuo algebra by a radical ideal, the radical itself being an ideal. Especially $A$ is the $\mathbb{F}$-span of the axes for the normal set.

This theorem contains a version of Sakuma's Theorem in the Jordan type context, since a primary step in its proof is to show that a 2-generated subalgebra is (essentially) of type $1 A, 2 B$, or $3 C(\eta)$, as is the case by definition in Matsuo algebras.

Corollary 8.4. Let $A$ be a primitive axial algebra of Jordan type $\eta \neq \frac{1}{2}$. Then $A$ is a quotient of the $\mathbb{F}$-permutation module for a set of 3-transpositions that is normal in its automorphism group. 
This generalizes observations of Miyamoto $\left(\eta=\frac{1}{4}\right.$, Ising $\sigma$-case $)$ and Shpectorov $\left(\eta=\frac{1}{32}\right.$, Ising $\tau$-case).

Cuypers and Hall [10] proved that all 3-transposition groups are locally finite. This implies

Corollary 8.5. A finitely generated primitive axial algebra of Jordan type $\eta \neq \frac{1}{2}$ is finite dimensional.

\section{Compact Axial Algebras of Jordan Type $\eta \neq \frac{1}{2}$}

Let us say that an axial algebra is compact if it is real and admits a symmetric form that is both invariant and positive definite. As we have mentioned above, compact Griess algebras and their generalizations due to Ivanov are primitive compact axial algebras. We do not assume the Norton inequality, which however does hold in these examples. By Theorem 8.3 every compact axial algebra of Jordan type $\eta \neq \frac{1}{2}$ must be the perpendicular direct sum of quotients of Matsuo algebras of Fischer spaces by their radicals. We encounter two fundamental questions:

(a) Which Fischer spaces can arise?

(b) Which of these algebras are actually Griess algebras?

By [23, (7.1)] the invariant symmetric form on a primitive axial algebra of Jordan type $\eta \neq \frac{1}{2}$ with Jordan axis set $\mathcal{P}$ must satisfy, after rescaling:

$$
\begin{array}{rrr}
1 A: & \langle\langle i, i\rangle\rangle=1 & \text { for } i \in \mathcal{P} ; \\
3 C(\eta): & \langle\langle i, j\rangle\rangle=\frac{\eta}{2} & \text { for }\{i, j, k\} \in \mathcal{L} ; \\
2 B: & \langle\langle i, j\rangle\rangle=0 & \text { otherwise. }
\end{array}
$$

Thus the Gram matrix of the form is

$$
I+\frac{\eta}{2} H
$$

where $H$ is the adjacency matrix for the diagram of the corresponding normal set of 3-transpositions. For this form to be positive (semi-)definite the adjacency matrix must therefore have minimal eigenvalue greater than (or equal to) $-\frac{2}{\eta}$. The relevant Ising cases are: 
(i) For $\eta=\frac{1}{4}$ this is -8 and was considered by Kitazume and Miyamoto [32] and Matsuo [36, 37.

(ii) For $\eta=\frac{1}{32}$ this is -64 and was considered by Hall and Shpectorov.

The direct summands of a compact algebra of Jordan type $\eta \neq \frac{1}{2}$ are primitive such algebras. Each of these is the quotient of a semi-definite Matsuo algebra by its radical and so is uniquely determined by its Fischer space and diagram. The first question above is thus reduced to finding the minimum eigenvalue of the diagram for a conjugacy class of 3-transpositions.

Theorem 9.1 (Hall and Shpectorov 2012). Let $r \geq 2$ be a positive integer. The diagram of a finite conjugacy class of 3-transpositions has minimal eigenvalue $\rho_{\min } \geq-r$ when the group is one of:

(a) infinitely many groups $3^{m} \rtimes 2\left(\right.$ where $\left.\rho_{\min }=-1\right)$;

(b) $m(r)$ distinct groups $N \rtimes \operatorname{Sym}(n)$ for each $n \geq 4$;

(c) $s(r)$ "sporadic" examples.

The functions $m$ and $s$, defined in the theorem and nondecreasing on $\mathbb{Z}_{\geq 2}$, are in general hard to calculate. For $\rho_{\min } \geq-8$ we have

$$
m(8)=4 \quad \text { and } \quad s(8)=12 \text {, }
$$

while for $\rho_{\min } \geq-64$ we have

$$
m(64)=13 \quad \text { and } \quad s(64)=90 .
$$

The compact Griess algebras of Jordan type $\eta \neq \frac{1}{2}$ must be primitive and compact axial algebras, but the converse need not be true. Let $m^{G}(r)$ and $s^{G}(r)$ be the functions that correspond to those of the theorem, counting respectively the number of extended symmetric and sporadic Griess algebra diagrams with minimal eigenvalue greater than or equal to $-r$.

For the Griess algebras that are the positive definite quotients of Matsuo algebras with $\rho_{\min } \geq-8$, Matsuo [36, 37] found that there are three infinite families and nine isolated examples. These are accounted for by:

(a) $m^{G}(8)=3$ :

$$
\mathrm{W}\left(A_{n}\right), \mathrm{W}\left(D_{n}\right)\left(=\mathrm{W}_{2}\left(\tilde{A}_{n-1}\right)\right), \mathrm{W}_{2}\left(\tilde{D}_{n}\right) ;
$$


(b) $s^{G}(8)=9$ :

$$
\mathrm{W}\left(E_{n}\right), \mathrm{W}_{2}\left(\tilde{E}_{n}\right) \text { with } n \in\{6,7,8\}, \mathrm{O}_{8}^{-}(2) \leq \operatorname{Sp}_{8}(2) \leq \mathrm{O}_{10}^{+}(2)
$$

The corresponding Griess algebra lists for $\rho_{\min } \geq-64$ are unknown.

The algebras with $\rho_{\text {min }} \geq-8$ in the gap between primitive compact axial and compact Griess are the algebras for

$$
3^{m} \rtimes 2, \mathrm{~W}_{3}\left(\tilde{A}_{n-1}\right), \mathrm{SU}_{4}(2), \mathrm{SU}_{5}(2), \mathrm{O}_{6}^{-}(3) .
$$

These all contain the $\mathrm{W}_{3}\left(\tilde{A}_{2}\right)=\mathrm{SU}_{3}(2)$ algebra - the Matsuo algebra for the affine plane of order 3 - and so are eliminated by:

Theorem 9.2 (Matsuo [36, 37]). For a compact Griess algebra of Jordan type $\eta=\frac{1}{4}$ the associated Fischer space is of symplectic type.

Matsuo proved this using

Theorem 9.3 (Miyamoto [40]). In a compact Griess algebra every idempotent is a conformal axis.

That is, it is ad-semisimple with a highly restricted fusion table arising from the representation theory of a related Virasoro algebra. On the other hand, any 3-transposition subgroup readily gives rise to idempotents of the corresponding parent Matsuo algebra. Matsuo's proof of Theorem 9.2 used this to show that there can be no transposition subgroups of type $\mathrm{SU}_{3}(2)$.

\section{Related Topics and Questions}

(a) In Griess' terminology, the twenty-six finite simple sporadic groups are partitioned as the "Happy Family" - the twenty sporadic groups involved in the Monster - and the "Pariahs" - the six not found within the Monster. (See [18] for further discussion.) The groups involved in the Monster act on $M^{\natural}$ and smaller algebras derived from it (although not all are generated by $2 A$ involutions), but more direct constructions would be of help in their study. For instance, Ryba [46] gave a construction, in the spirit of Norton [41], of a commutative $\mathbb{F}_{2}$-algebra of dimension 4370 admitting the Baby Monster. As already mentioned, there is hope for direct construction of the transposition algebras in characteristic 2 
for $F i_{23}$ and $3 \cdot F i_{24}$ of respective dimensions 782 and 783 . The Fischer group $F i_{23}$ is a subgroup of the Baby Monster, and Ryba observed the 782 algebra as an $\mathrm{Fi}_{23}$-invariant direct factor in the 4370 algebra.

(b) Perhaps more interesting would be the construction of nice algebras for the pariahs. Frohardt [14] emulated Griess [17] in constructing a commutative algebra of dimension 85 that admits the Janko pariah $J_{3}$ as an automorphism group. In 15] he outlined a similar procedure for constructing an algebra of dimension 495 that admits the triple cover of the O'Nan simple group. In that paper, he also discussed candidates for two other pariahs - a commutative algebra of dimension 48174 for the Lyons group and a noncommutative algebra of dimension 887778 for the largest Janko group $J_{4}$. The smallest Janko pariah $J_{1}$ is a subgroup of O'Nan and also of $\mathrm{G}_{2}(11)$. The final pariah, the Rudvalis group, has a double cover contained in $\mathrm{E}_{7}(5)$.

(c) Rehren [44, 45] has studied the more general fusion rules of Ising type $\mathcal{I}_{\alpha, \beta}$ given by the fusion table:

\begin{tabular}{cc||ccc|c} 
& & & + & & - \\
& $\mathcal{I}_{\alpha, \beta}$ & 1 & 0 & $\alpha$ & $\beta$ \\
\hline \hline & 1 & 1 & $\emptyset$ & $\alpha$ & $\beta$ \\
+ & 0 & $\emptyset$ & 0 & $\alpha$ & $\beta$ \\
& $\alpha$ & $\alpha$ & $\alpha$ & 1,0 & $\beta$ \\
\hline- & $\beta$ & $\beta$ & $\beta$ & $\beta$ & $1,0, \alpha$
\end{tabular}

Except in degenerate situations, nontrivial Miyamoto involutions exist. A central question is the extent to which a version of Sakuma's Theorem can be proven. Rehren makes some progress but a final answer is not clear. Conversely, by looking at "axial covers" of the standard dihedralalgebras, he details which of the Conway-Norton-Sakuma algebras have natural generalizations for parameter sets $(\alpha, \beta)$ other than $\left(\frac{1}{4}, \frac{1}{32}\right)$.

(d) Vertex operator algebras and Griess algebras are defined in characteristic 0. Rehren's work on axial covers (mentioned above) and work of Simon [50] on $\mathbb{Z}$-forms of various Griess algebras speak to a change of the ring of constants, but appropriate general definitions are not clear. Furthermore, these treatments always require 2 to be invertible, whereas 
the algebras of Cuypers, Horn, in 't panhuis, Shpectorov [9], Ryba [46], and various other algebras of interest are defined in characteristic 2 .

(e) Since it is the weight 2 piece of a vertex operator algebra, a Griess algebra has finite dimension. But in Corollary 8.5 we saw that finitely generated primitive axial algebras of Jordan type $\eta \neq \frac{1}{2}$ can be proven to be finite dimensional. The same is true of Kaplansky's Lie algebras. What conditions on a triple algebra or axial algebra force this? Or, conversely, is there benefit in assuming a finitely generated locally defined algebra to be finite dimensional?

(f) Axial algebras of Jordan type $\eta=\frac{1}{2}$ have been studied extensively $[24$, 25], but their structure and classification are still not complete or evident.

(g) Results of Miyamoto [40] force compact Griess algebras of Ising type to be primitive as axial algebras. What is the structure of imprimitive axial algebras of the types studied so far? In particular, can their study be reduced to the primitive case?

(h) Griess algebras possess many properties that are not assumed for axial algebras. What kind of progress can be made by taking on these or similar properties? What about the assumption of an invariant form? positive (semi-)definite? satisfying the Norton inequality?

(i) Miyamoto showed that all idempotents of Griess algebras are multiples of conformal vectors. That result bears fruit, as we saw in Theorems 9.2 and 9.3 above. This suggests that careful study of idempotents in axial and related (e.g., Matsuo) algebras is worthwhile. Rehren has pursued this in [42, 43, 45]. As mentioned above, subspaces of Fischer spaces readily give rise to idempotents in the associated Matsuo and axial algebras.

(j) What can be said about an LN algebra (or transposition algebra) in which all the line-algebras (dihedral-algebras) are from the ConwayNorton-Sakuma list or Rehren's generalizations? The same question can be asked for Griess algebras.

(k) When is a real compact axial algebra a Griess algebra?

(l) While Matsuo determined precisely the compact Griess algebras that occur with Jordan type $\eta=\frac{1}{4}$ (the Ising $\sigma$-case), the more difficult $\eta=\frac{1}{32}$ (the Ising $\tau$-case) remains open. A result of Chen and Lam [6] shows that the corresponding 3 -transposition groups need not be of symplectic type. Non-Ising fusion also provides Jordan type examples. For instance, Lam and Yamauchi 33] constructed Griess algebras of Jordan type $\eta=\frac{1}{5}$, 
including one associated with the 3 -transposition group $\mathrm{O}_{8}^{-}(3) .9$ Still further relationships among 3-transposition groups, Griess algebras, and vertex operator algebras are given by Lam and Yamauchi 34]. Whether these connections are coincidental or part of a deeper relationship is unclear.

(m) A reason the link between algebras and 3-transposition groups has been fruitful is that these groups are essentially classified [11, 10]. But the 6-transposition Monster and its related algebras suggest that any question asked regarding 3-transposition groups might be of interest when extended to 4-transposition groups, 5-transposition groups, or even 6transposition groups - cases where classification results are thin on the ground. Interesting subclasses of groups would correspond to interesting classes of algebras.

\section{References}

1. R. Block, New simple Lie algebras of prime characteristic, Trans. Amer. Math. Soc. 89 (1958), 421-449.

2. R. E. Borcherds, Vertex algebras, Kac-Moody algebras, and the Monster, Proc. Nat. Acad. Sci. U.S.A., 83 (1986), 3068-3071.

3. A. E. Brouwer, A. M. Cohen, H. Cuypers, J. I. Hall and E. Postma, Lie algebras, 2-groups and cotriangular spaces, Adv. Geom., 12 (2012), 1-17

4. F. Buekenhout, La géométrie des groupes de Fischer, Free University of Brussels, unpublished notes, 1974.

5. P. J. Cameron, J. M. Goethals and J. J. Seidel, The Krein condition, spherical designs, Norton algebras and permutation groups, Nederl. Akad. Wetensch. Indag. Math., 40 (1978), 196-206.

6. H. -Y. Chen and C. H. Lam, An explicit Majorana representation of the group $3^{2}: 2$ of 3C-pure type, Pacific J. Math. 271 (2014), 25-51.

7. J. H. Conway, A simple construction for the Fischer-Griess monster group, Invent. Math., 79 (1985), 513-540.

8. H. Cuypers, Lie algebras and cotriangular spaces, Bull. Belg. Math. Soc. Simon Stevin, 12 (2005), 209-221.

9. H. Cuypers, M. Horn, J. in 't panhuis and S. Shpectorov, Lie algebras and 3-transpositions, J. Algebra, 368 (2012), 21-39.

\footnotetext{
${ }^{9}$ The 11073 -transpositions (axes) have diagram with minimal eigenvalue $-10=-2 / \eta$, hence its Matsuo algebra is positive semi-definite. The positive definite quotient algebra has dimension 288.
} 
10. H. Cuypers and J. I. Hall, The 3-transposition groups with trivial center, J. Algebra, 178 (1995), 149-193.

11. B. Fischer, Finite groups generated by 3-transpositions I, Invent. Math., 13 (1971), 232-246.

12. I. B. Frenkel, J. Lepowsky and A. A. Meurman, A natural representation of the Fischer-Griess Monster with the modular function $J$ as character, Proc. Nat. Acad. Sci. U.S.A., 81 (1984), 3256-3260.

13. I. B. Frenkel, J. Lepowsky and A. A. Meurman, "Vertex Operator Algebras and the Monster," Pure and Applied Mathematics, 134, Academic Press, Inc., Boston, MA, 1988.

14. D. Frohardt, A trilinear form for the third Janko group, J. Algebra, 83 (1983), 349-379.

15. D. Frohardt, Toward the construction of an algebra for O'Nan's group, "Proceedings of the Rutgers Group Theory Year, 1983-1984," Cambridge Univ. Press, Cambridge, 1985, 107-110.

16. R. L. Griess, Jr., A construction of $F_{1}$ as automorphisms of a 196,883-dimensional algebra, Proc. Nat. Acad. Sci. U.S.A., 78 (1981), 686-691.

17. R. L. Griess, Jr., The friendly giant, Invent. Math., 69 (1982), 1-102.

18. R. L. Griess, Jr., "Twelve Sporadic Groups," Springer Monographs in Mathematics, Springer-Verlag, Berlin, 1998.

19. J. I. Hall, Graphs, geometry, 3 -transpositions, and symplectic $\mathbb{F}_{2}$-transvection groups, Proc. London Math. Soc. (Series 3), 58 (1989), 89-111.

20. J. I. Hall, Some 3-transposition groups with normal 2-subgroups, Proc. London Math. Soc. (Series 3), 58 (1989), 112-136.

21. J. I. Hall, "Moufang Loops and Groups with Triality are Essentially the Same Thing," Memoirs of the American Mathematical Society, to appear.

22. J. I. Hall, F. Rehren and S. Shpectorov, Universal axial algebras and a theorem of Sakuma, J. Algebra, 421 (2015), 394-424.

23. J. I. Hall, F. Rehren and S. Shpectorov, Primitive axial algebras of Jordan type, J. Algebra, 437 (2015), 79-115.

24. J. I. Hall, Y. Segev and S. Shpectorov, Miyamoto involutions in axial algebras of Jordan type half, Israeli J. Math., to appear.

25. J. I. Hall, Y. Segev and S. Shpectorov, On primitive axial algebras of Jordan type, submitted.

26. R. C. Hamelink, Lie algebras of characteristic 2, Trans. Amer. Math. Soc., 144 (1969), 217-233.

27. D. G. Higman, Finite permutation groups of rank 3, Math. Z., 86 (1964), 145-156.

28. A. A. Ivanov, "The Monster Group and Majorana Involutions," Cambridge Tracts in Mathematics 176, Cambridge University Press, Cambridge, 2009. 
29. A. A. Ivanov, D. V. Pasechnik, Á. Seress, S. Shpectorov, Majorana representations of the symmetric group of degree 4, J. Algebra, $\mathbf{3 2 4}$ (2010), 2432-2463.

30. N. Jacobson, "Structure and Representations of Jordan Algebras," American Mathematical Society Colloquium Publications 39, American Mathematical Society, Providence, R. I., 1968.

31. I. Kaplansky, Some simple Lie algebras of characteristic 2, in: "Lie Algebras and Related Topics (New Brunswick, N. J., 1981)," Lecture Notes in Math. 933, Springer, BerlinNew York, 1982, 127-129.

32. M. Kitazume and M. Miyamoto, 3-transposition automorphism groups of $V O A$ "Groups and Combinatorics-in Memory of Michio Suzuki," Adv. Stud. Pure Math., 32, Math. Soc. Japan, Tokyo, 2001, 315-324.

33. C. H. Lam and H. Yamauchi, On 3-transposition groups generated by $\sigma$-involutions associated to $c=4 / 5$ Virasoro vectors, J. Algebra 416 (2014), 84-121.

34. C. H. Lam and H. Yamauchi, The Conway-Miyamoto correspondences for the Fischer 3-transposition groups, preprint, April 2016.

35. H. S. Li, Symmetric invariant bilinear forms on vertex operator algebras, J. Pure Appl. Algebra, 96 (1994), 279-297.

36. A. Matsuo, 3-transposition groups of symplectic type and vertex operator algebras (version 1), manuscript, November 2003. (available as arXiv. math/0311400v1)

37. A. Matsuo, 3-transposition groups of symplectic type and vertex operator algebras, $J$. Math. Soc. Japan, 57 (2005), 639-649.

38. A. Matsuo and M. Matsuo, On a commutative nonassociative algebra associated with $\mathrm{GL}_{3}(2)$, manuscript, August 1999.

39. J. McLaughlin, Some subgroups of $\mathrm{SL}_{n}\left(F_{2}\right)$, Illinois J. Math., 13 (1969), 108-115.

40. M. Miyamoto, Griess algebras and conformal vectors in vertex operator algebras, J. Algebra, 179 (1996), 523-548.

41. S. Norton, On the group $F i_{24}$, Geom. Ded., 25 (1988), 483-501.

42. F. Rehren, Linear idempotents in Matsuo algebras, Indiana Univ. Math. J., 65 (2016), 1713-1733.

43. F. Rehren, Fusion rules from root systems I: case $A_{n}$, preprint, 13 March 2014, (available as arXiv. math/1403. 3308).

44. F. Rehren, Generalised dihedral subalgebras from the Monster, preprint, 10 October 2014, (available as arXiv. math/1510. 00640).

45. F. Rehren, "Axial Algebras," Ph.D. Thesis, University of Birmingham, 28 February 2015, 156 pages.

46. A. J. E. Ryba, A natural invariant algebra for the Baby Monster group, J. Group Theory 10 (2007), 55-69. 
47. S. Sakuma, 6-transposition property of $\tau$-involutions of vertex operator algebras, Int. Math. Res. Not. IMRN 2007, no. 9, Art. ID rnm 030.

48. L. L. Scott, Some properties of character products, American Mathematical Society Colloquium Publications, J. Algebra, 45 (1977), 259-265.

49. E. E. Shult, Groups, polar spaces and related structures, in: "Proceedings of the NATO Advanced Study Institute Held at Nijenrode Castle, Breukelen, 8-20 July 1974," eds. : M. Hall, Jr., and J. H. van Lint, NATO Advanced Study Institutes Series, Series C: Mathematical and Physical Sciences 16, D. Reidel Publishing Co., Dordrecht-Boston, Mass., Mathematical Centre, Amsterdam, 1974.

50. G. G. Simon, "Automorphism-invariant Integral Forms in Griess Algebras," Ph.D. Thesis, University of Michigan, February 9, 2016, 141 pages. 